\title{
Early onset Cockayne's syndrome: case reports with neuropathological and fibroblast studies
}

\author{
M A PATTON*, F GIANNELLI $\dagger$, A J FRANCIS $\dagger$, M BARAITSER
} B HARDING $\ddagger$, AND A J WILLIAMS

From *St George's Hospital Medical School, London SW17; †Paediatric Research Unit, Guy's Hospital, London SE1; $¥$ Hospitals for Sick Children, Great Ormond Street, London WC1; and §Ysbyty Glan Clwyd, Bodelwyddan, Rhyl, $N$ Wales.

SUMmARY Two patients with early onset Cockayne's syndrome are presented. In each case there was a striking failure of growth and developmental deterioration around six months of age. $\frac{\vec{v}}{\mathrm{v}}$ It has been suggested that early onset Cockayne's syndrome is a syndrome dinstinct from $₫$

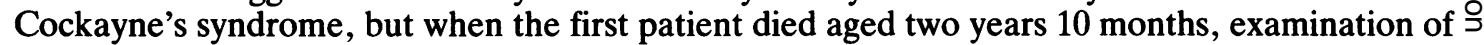
the brain showed a leucodystrophy with 'tigroid' demyelination similar to that reported in later $\vec{z}$ onset cases of Cockayne's syndrome. Studies of the effects of UV irradiation on cultured fibroblasts from patients showed similar levels of inhibition of RNA synthesis to those seen in a $\stackrel{\rho}{\Im}$ control with Cockayne's syndrome.

This evidence suggests it is appropriate to classify early onset Cockayne's syndrome with $\mathscr{\bigotimes}_{0}^{\infty}$ Cockayne's syndrome. Since there is a phenotypic overlap between early onset Cockayne's $\square$ syndrome and COFS syndrome, they may both be classified within the same diagnostic group, 咸 but as yet no cellular studies with UV irradiation have been performed in COFS syndrome.

Cockayne's syndrome is an autosomal recessive disorder with short stature, an atypical retinopathy, deafness, mental deterioration, skin sensitivity to ultraviolet light, and a characteristic facies with marked enophthalmos and a beaked nose. Other features include cataracts, joint contractures, and a peripheral neuropathy. Since it was first described in $1936^{1}$ it has become apparent that it is a progressive disorder with an underlying leucodystrophy.

In 1971 Lowry et al ${ }^{2}$ described what they felt to be a new syndrome in a brother and sister with cataracts, microcephaly, kyphosis, and joint contractures. The classification of this entity has been controversial. Sugarman ${ }^{3}$ suggested that this syndrome might be an early form of Cockayne's syndrome, while Pena et $a l^{4}$ suggested that the features resembled the cerebro-oculo-facial-skeletal (COFS) syndrome.

We present two unrelated patients with clinical features similar to those described by Lowry et al. ${ }^{2}$ In both, profound growth retardation developed within the first year of life and the facial features became identical to those of Cockayne's syndrome.

Received for publication 22 June 1988.

Revised version accepted for publication 26 August 1988.
There was also associated photosensitivity. UV irradiation studies on skin fibroblasts were carried out and showed an inhibition of RNA synthesis similar to that seen in the control with the later onset Cockayne's syndrome. The first patient died aged two years 10 months and necropsy of the brain showed a 'tigroid' pattern of demyelination similar to that reported in Cockayne's syndrome. ${ }^{5}$

\section{Case reports}

CASE 1

This boy was the second child of healthy, unrelated parents. At his birth his mother was 43 years and his $N$ father 22 years. His mother had two children by a previous marriage, the oldest of whom had died 0 from a pulmonary embolus. He was born at term $\mathrm{\omega}$ after a normal pregnancy, with a birthweight of ${ }^{0}$ $3640 \mathrm{~g}$ and head circumference $33.2 \mathrm{~cm}$. At birth a number of dysmorphic features were noted. He had $\mathbb{\varnothing}$ deep set eyes with bilateral cataracts, his palate was ? high, and his uvula was bifid. There were fixed $\frac{T}{T}$ flexion deformities of the fingers with the thumb $\underset{\mathbb{D}}{\circ}$ adducted across the palm. Hip movement was $\stackrel{?}{\odot}$ limited especially on the left and there were bilateral $\stackrel{\varnothing}{\varrho}$ rocker bottom feet. An initial diagnosis of trisomy 
18 was made, but the karyotype was normal. Other routine laboratory investigations were also normal.

In the first few months there were feeding problems because of oesophageal reflux, but he gained weight satisfactorily up to the age of seven months (fig 1). Thereafter his weight remained static, even with tube feeding, until it declined further in the terminal stages of the illness. After 10 months there was no further growth in the head circumference $(37.5 \mathrm{~cm})$.

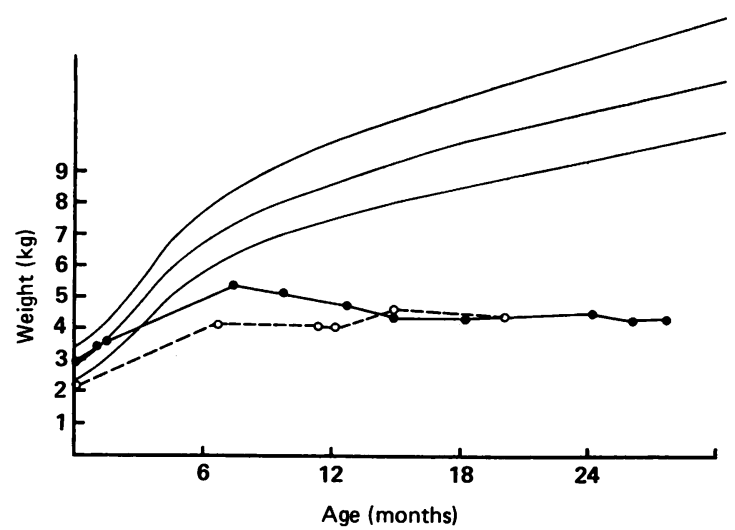

FIG 1 Growth charts (with 10th and 90th centile) showing lack of weight gain from around six months.

Patient $1=-$. Patient $2=--\mathrm{O}--$.

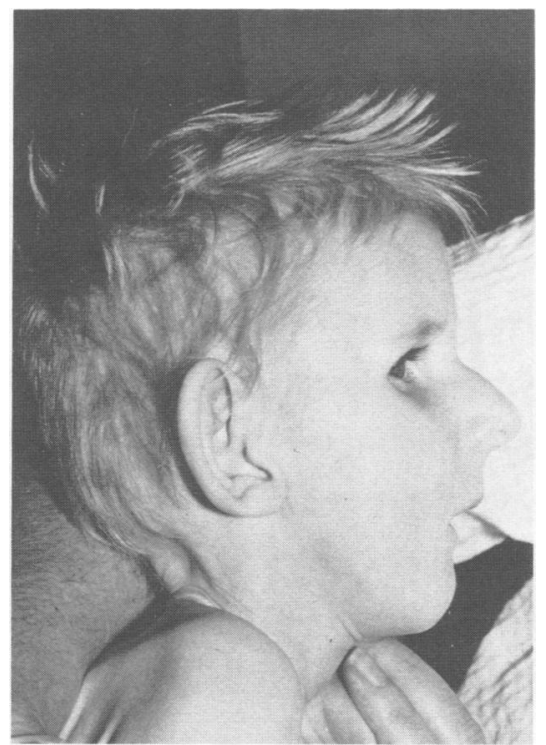

Between three and four months his cataracts were operated on and contact lenses provided. The contact lenses were poorly tolerated because of reduced lacrimal secretion. Postoperative examination of the fundus showed hypoplastic optic nerves and there was little evidence of useful vision.

In the second year he became very emaciated with kyphosis (fig 2). There was limitation of joint movement in the knees and he showed marked photosensitivity to sunlight. He was grossly retarded with a developmental age of four to five months. In the last months he suffered many chest infections. $\mathrm{He}$ died from a chest infection at two years 10 months.

A neuropathological examination showed a very small, symmetrical brain ( $335 \mathrm{~g}$, normal $1120 \mathrm{~g}$ ) with the hind brain accounting for only $5 \%$ of the total (normal 12\%). Coronal slices showed normal cortical ribbon and basal ganglia, shrunken thalamus, flattened hippocampus, greatly dilated ventricles, and much reduced white matter with a strikingly stripy or tigroid appearance (fig 3 ). The optic nerves were thin and grey.

Histology confirmed the severe though discontinuous demyelination and concomitant gliosis throughout the neuraxis. In addition the cerebral

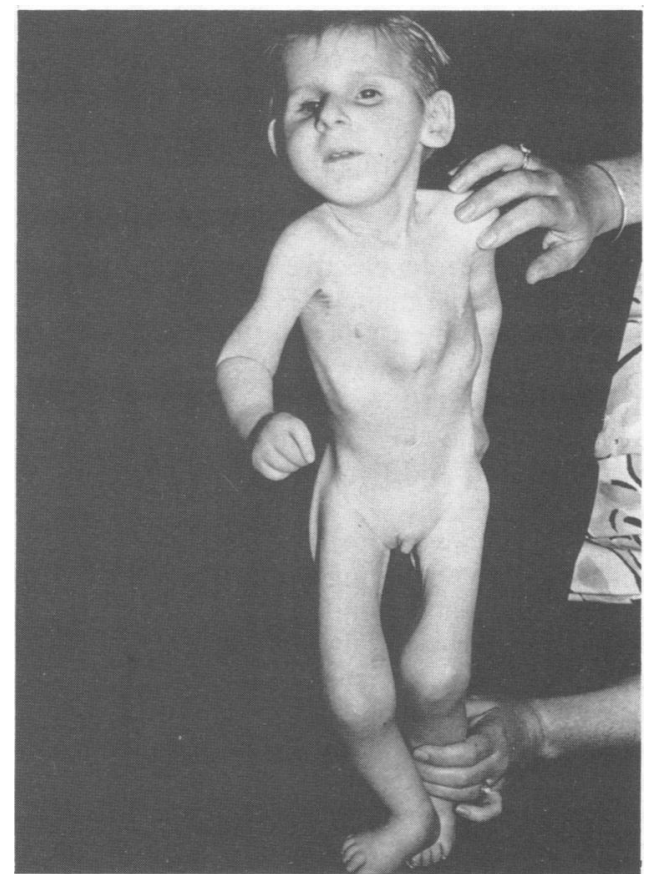

FIG 2 Case 1 at 18 months. Cachectic appearance is now very striking. Marked kyphosis and joint contractures also present. 


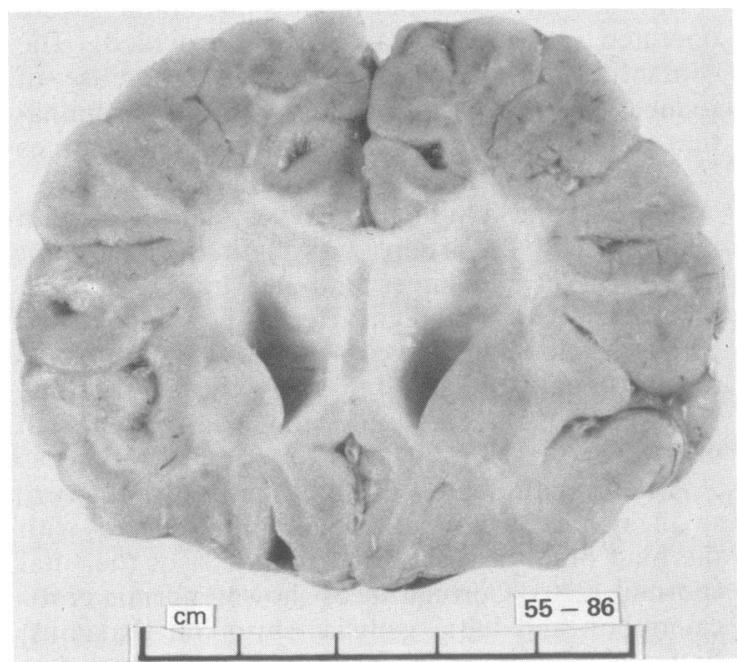

FIG 3 Cut section of the brain from case 1 showing ventricular enlargement, cerebral atrophy, and reduced white matter.

cortex, basal ganglia, and thalamus contained multitudes of mulberry-like calcific concretions. Calcifications were also present in the walls of meningeal blood vessels and very commonly in the cerebellar cortex where Purkinje cell somata and dendritic trees were mineralised, and there were marked degenerative changes with axonal torpedoes and dendritic asteroid formations.

\section{CASE 2}

This girl was born by elective caesarian section after an uneventful pregnancy. Her parents were first cousins. She weighed $2700 \mathrm{~g}$ at birth and there were no neonatal problems. The parents first became worried at about 10 weeks when they noticed that she was not following or fixing. Bilateral cataracts were diagnosed and lens extractions carried out at four months.

Her general development seemed slow and by six months she was not sitting alone. She seemed listless and apathetic and tended to fall asleep while feeding. On examination her weight, length, and head circumference were well below the 3rd centile. She was clearly retarded and microcephalic with microphthalmia and a pendular searching nystagmus. Her liver was moderately enlarged. $X$ ray of the hips showed mild bilateral subluxation of the hips and shallow acetabulae. A CT brain scan showed mild cerebral atrophy, but no calcification was seen. An EMG showed some evidence of partial denervation suggestive of anterior horn cell involve- ment. Because of the combination of microcephaly, cataract, beakish nose, and small jaw a tentative diagnosis of the COFS syndrome was made.

There was persistent vomiting and a Nissen fundoplication and gastrostomy were carried out. Her weight remained static and she was readmitted at the age of nine months. Further investigations were performed including chromosome studies, immunoglobulins, $\mathrm{T}$ cells subsets, neutrophil mobility, and a bone marrow examination. All of these were normal. Liver biopsy showed normal architecture.

On examination at that stage her weight was 4.96 $\mathrm{kg}$ (well below the 7th centile and a slight reduction from five months previously). Her length was $78 \mathrm{~cm}$ and the head circumference was $38.2 \mathrm{~cm}$. The eyes had become more sunken (fig 4). She had a narrow face with an appearance of sunburnt cheeks. There was a generalised increase in tone. Contractures of the hip adductors had developed bilaterally.

A developmental review showed that there had been little progress in terms of manipulation, vision, and speech. It was noted that her hearing seemed acute. At that stage her facial features were very suggestive of the early onset Cockayne's syndrome. A skin biopsy to look at photosensitivity of the fibroblasts was performed.

\section{CELLULAR STUDIES}

In Cockayne's syndrome there is marked cellular sensitivity to UV irradiation manifesting as reduced survival $^{6}$ and failure of cultured cells to recover a

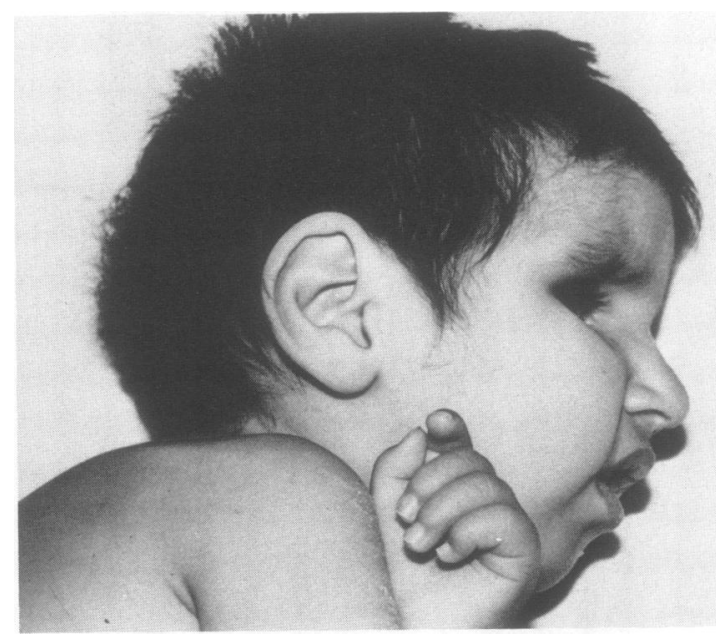

FIG 4 Case 2 at nine months. The microcephaly and enophthalmos had initially suggested a diagnosis of COFS syndrome. 

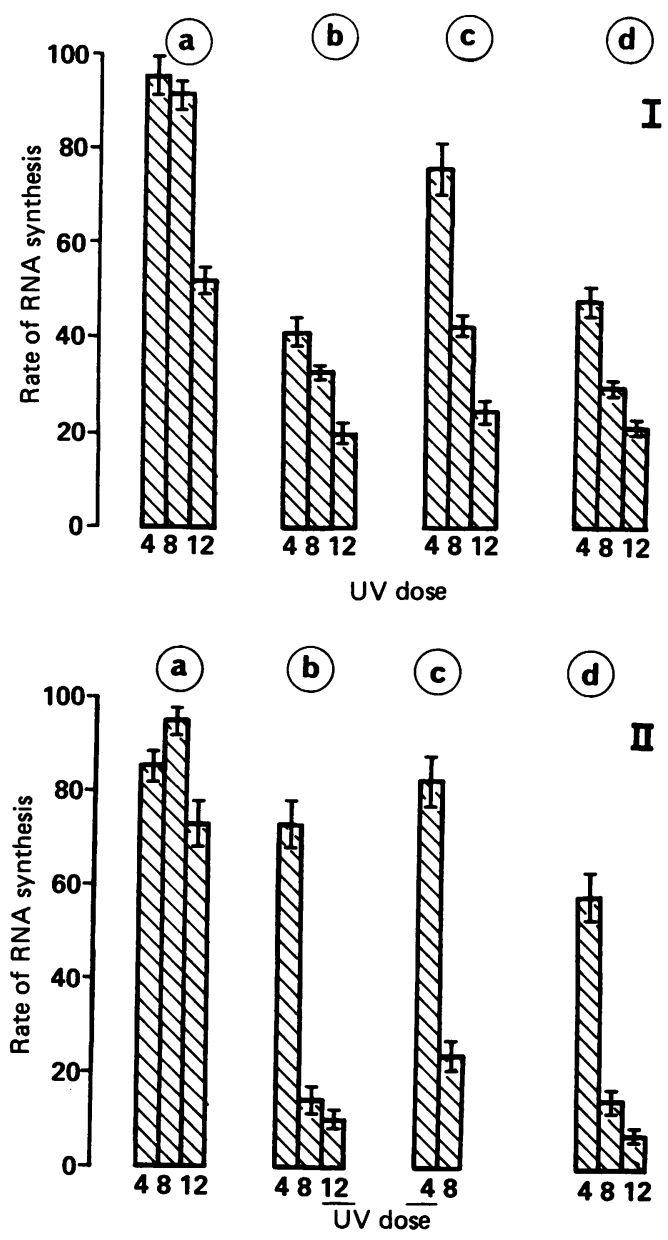

FIG 5 Rate of RNA synthesis in irradiated G1 and G2 phase cells expressed as a percentage of the rate of sham irradiated control cells (samples of at least 25 cells were analysed for each experimental point), I 24 hours after irradiation, and II 48 hours after irradiation: $(a)=$ average of two normal control strains, $(b)=$ Cockayne's syndrome control, $(c)=$ patient $1,(d)=$ patient 2 . UV dose is in J.m-2. Vertical lines in histograms $=S E M$.

normal rate of DNA and RNA synthesis several hours after irradiation. ${ }^{78}$ Such a cellular response has proved consistent enough to be used in the postnatal and prenatal diagnosis of the disease. ${ }^{9}$ For this reason, and because of the clinical evidence of photosensitivity, it was felt that fibroblast strains from the patients described above should be tested for their response to UV irradiation. Skin biopsies were obtained and fibroblast strains were established in DMEM medium supplemented with $10 \%$ calf serum, penicillin, and streptomycin. These strains plus two normal and one Cockayne's syndrome control strain were irradiated with $0,4,8$, and $12 \mathrm{~J} . \mathrm{m}$ of germicidal UV light in order to measure their recovery from inhibition of RNA synthesis 24 and 48 hours after irradiation. This was done by the autoradiographic assay previously described. ${ }^{9}$ Briefly, cells plated on coverslips and UV irradiated were pulse laballed for one hour with $5 \mu \mathrm{Ci} / \mathrm{ml}{ }^{3} \mathrm{H}$-uridine $(25 \mathrm{Ci} / \mathrm{mmol})$ and for three hours after irradiation with $10 \mu \mathrm{Ci} / \mathrm{ml}^{3} \mathrm{H}$-thymidine $(25 \mathrm{Ci} / \mathrm{mmol}) 3,6,24$, and 48 hours after irradiation. Then they were fixed and coated with autoradiographic film for a 24 hour exposure. After development, cells undergoing DNA replication were easily identified by their dense labelling and excluded from the analysis. The remaining cells that were in the $\mathrm{G} 1$ and G2 phase of the cell cycle undertook only RNA synthesis, and this was measured by counting the autoradiographic grains overlapping the cells.

This analysis showed a 40 to $60 \%$ inhibition of RNA synthesis in all irradiated strains at three to six hours after irradiation (data not shown). However, at 24 and 48 hours after irradiation the rate of RNA synthesis had recovered in normal cells exposed to all but the highest dose (fig 5a). By contrast, the Cockayne's syndrome control showed further decrease of RNA synthesis (fig 5b). The fibroblasts of patient 1 failed to recover adequately (fig 5c) and those of patient 2 behaved in an identical manner to the Cockayne's syndrome control (fig 5d).

\section{Discussion}

In 1971 Lowry et $a l^{2}$ described a brother and sister with bilateral congenital cataracts, microcephaly, progressive joint contractures, and kyphosis. In both cases the facial features were similar with large ears, enophthalmos, and a prominent nasal bridge. In both patients there was very marked growth delay. Their first patient weighed $4.8 \mathrm{~kg}$ at five years and their second patient weighed $7.6 \mathrm{~kg}$ at seven years of age. Both children were severely retarded. A necropsy with a full neuropathological examination was available from the second patient. ${ }^{10}$ This showed a very small brain with atrophy of the white matter. There was extensive calcification in both the cerebrum and cerebellum. The retina showed pigmentary changes. Although many of the clinical and pathological features were similar to those seen in Cockayne's syndrome, the authors felt that the early onset distinguished this disorder from the typical Cockayne's syndrome.

Since then two further reports of early onset Cockayne's syndrome have been published. Moyer et $a^{11}$ reported monozygous twins with similar 
growth failure. The maximum recorded weight was $4 \mathrm{~kg}$ at 12 months and thereafter there was no further weight gain. Cataracts were not present at birth, but had developed at two and a half years in one of the twins. The other report by Houston et al ${ }^{12}$ was of monozygous twins and an affected brother. Once again the growth failure started in the first year of life and very little growth took place after this. Skin photosensitivity was reported as a prominent feature in one of the twins. The neuropathological findings reported in these two papers are similar to those reported by Dolman et al ${ }^{10}$ and are comparable with the findings in Cockayne's syndrome. ${ }^{5}$

There are many similarities between COFS syndrome and early onset Cockayne's syndrome. When COFS syndrome was first described by Pena and Shokeir ${ }^{13}$ in 1974 , they reported the features of microcephaly, microphthalmia, cataracts, blepharophimosis, flexion contractures, skeletal abnormalities, and failure to thrive in infants between the ages of one month and 27 months. Most of the patients in their series died in infancy with a mean age of death of 13.7 months. However, in 1978 Pena et $a l^{4}$ reviewed two of the surviving patients and found a third member of the family had become affected. One had lived until five years by which time she was profoundly cachectic and had not increased her weight from five months of age $(3.3 \mathrm{~kg})$. At necropsy there were small foci of calcific deposits in both the grey and white matter and there was also a depletion of white matter compatible with a leucodystrophy. Their second patient was institutionalised when examined at three and a half years of age, and had not gained weight despite adequate caloric intake. His weight was $4 \cdot 3 \mathrm{~kg}$. Clinically he was microcephalic with deep set eyes, cataracts, a prominent nasal bridge, and flexion contractures at the elbows and knees. Their third patient was seen at four years of age and weighed $3.6 \mathrm{~kg}$. He had had similar problems and facially resembled the appearance seen in Cockayne's syndrome.

The growth pattern of the two patients in this report has been striking. Both had normal birth weights and appeared to grow normally for the first few months of life, but from around six months of age showed virtually no further weight gain. Increase in length also virtually ceased at this stage, but this was more difficult to quantify because of the joint contractures. There were considerable feeding problems but these were unlikely to be the most significant factor in the growth failure. The first patient was the subject of a local newspaper article describing him as "the boy who wouldn't grow". The striking failure to thrive with subsequent emaciation is similar to that seen in the reported cases of both early onset Cockayne's syndrome and COFS syndrome.

Both patients in this report showed severe de-o velopmental delay. In the neonatal period there was 흐 hypotonia and later spastic quadriplegia developed. $\frac{\bar{s}}{\bar{D}}$ Neither children acquired speech or sat indepen- $\stackrel{\mathbb{Q}}{\Omega}$ dently. The CT brain scans in the second patiento showed generalised cerebral atrophy but no evi- dence of calcification. The neuropathological ex- $\cdot \vec{\circ}$ amination in patient 1 showed reduction in white $\overrightarrow{\vec{\omega}}$ matter with demyelination in a pattern consistent $\stackrel{\omega}{\mathscr{S}}$ with the leucodystrophy seen in Cockayne's syndrome.

The patients reported here had various degrees of skin photosensitivity, which led us to investigate the $\omega$ effects of germicidal UV light on the fibroblasts. $\vec{T}$ The level of RNA synthesis after UV light was inhibited at 24 and 48 hours in both patients. In the second patient the response was almost identical to $\overrightarrow{\vec{z}}$ that seen in the Cockayne's syndrome control.

The combination of clinical features, neuro- $\bar{a}$ pathology, and fibroblast studies indicates that the $\vec{\theta}$ early onset Cockayne's syndrome is correctly classi- $\infty$ fied with Cockayne's syndrome. The underlying. biochemical abnormality in Cockayne's syndrome has not been identified, and there may still be biochemical differences between the early onseto Cockayne's syndrome and that with a later onset. Fibroblast complementation studies have indicated $\stackrel{\mathbb{Q}}{\mathscr{Q}}$ that three complementation groups exist. ${ }^{14}$ Comple- $\vec{\Rightarrow}$ mentation studies have not as yet been undertaken 3 in the patients in this report.

It would also seem appropriate to consider COFS syndrome within the same classification. The natural history and neuropathology reported by Pena et al $l^{4}$ has considerable similarity with the cases reported here. No studies on the effects of UV irradiation on: fibroblasts from patients with COFS syndrome haveo̊ been reported.

The cellular studies were supported by a grant from the Cancer Research Campaign. Secretarial assistance was provided by Mrs Sheena Willoughby.

\section{References}

1 Cockayne EA. Dwarfism with retinal atrophy and deafness $\omega$ Arch Dis Child 1936;11:1-8.

2 Lowry RB, MacLean R, McLean DM, Tischler B. Cataracts microcephaly, kyphosis and limited joint movement in two siblings: a new syndrome. J Pediatr 1971;79:282-4.

3 Sugarman GI. Syndrome of microcephaly, cataracts, kyphosis, and joint contractures versus Cockayne syndrome. J Pediatr 1973;82:351.

4 Pena SDJ, Evans J, Hunter AGW. COFS syndrome revisited. Birth Defects 1978;XIV(6B):205-13.

5 Moosy J. The neuropathology of Cockayne's syndrome. J Neuropathol Exp Neurol 1967;26:654-60.

6 Wade MH, Chu EHY. Effects of DNA damaging agents on

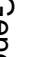


cultured fibroblasts derived from patients with Cockayne syndrome. Mutat Res 1979;59:49-60.

7 Lehmann AR, Kirk-Bell S, Mayne L. Abnormal kinetics of DNA synthesis in ultraviolet-irradiated cells from patients with Cockayne's syndrome. Cancer Res 1979;39:4237-41.

${ }^{8}$ Mayne LV, Lehmann AR. Failure of RNA synthesis to recover after UV-irradiation: an early defect in cells from individuals with Cockayne's syndrome and xeroderma pigmentosum. Cancer Res 1979;42:1473-8.

9 Lehmann AR, Francis AJ, Giannelli F. Prenatal diagnosis of Cockayne's syndrome. Lancet $1985 ; \mathbf{i}: 486-8$.

${ }_{10}$ Dolman CL, Wright VJ. Necropsy of original case of Lowry's syndrome. J Med Genet 1978;15:227-45.

11 Moyer DB, Marquis P, Shertzer ME, Burton BK. Cockayne syndrome with early onset of manifestations. Am J Med Genet 1982;13:225-30.

12 Houston CS, Zaleski WA, Rozdilsky B. Identical male twins and brother with Cockayne syndrome. Am J Med Genet 1982;13:211-23.

13 Pena SDJ, Shokeir MHK. Autosomal recessive cerebro-oculofacial-skeletal (COFS) syndrome. Clin Genet 1974;5:285-93.

${ }^{14}$ Lehmann AR. Three complementation groups in Cockayne syndrome. Mutat Res 1982;106:347-56.

Correspondence to Dr M A Patton, Department of Clinical Genetics, St George's Hospital Medical School, Cranmer Terrace, London SW17 0RE. 